This is the author's copy of the publication as archived with the DLR's electronic library at http://elib.dlr.de. Please consult the original publication for citation.

\title{
Worst Case Analysis of a Saturated Gust Loads Alleviation System
}

\author{
Andreas Knoblach and Harald Pfifer and Peter Seiler
}

In order to determine a guaranteed upper bound for worst case 1-cosine gust loads of flexible aircrafts, the usage of the worst case energy-to-peak gain of linear parameter-varying (LPV) systems has been recently proposed. A limitation of this approach is that it cannot deal with nonlinearities. This paper uses integral quadratic constraints (IQCs) to circumvent this restriction and to consider the saturation of a gust loads alleviation system. Based on the dissipation inequality framework, a linear matrix inequality constraint which bounds the worst case energy-to-peak gain of saturated LPV systems is given. In order to reduce the conservatism, an iterative procedure to refine local IQCs is proposed. The conservatism of this approach is analyzed at the example of a two-dimensional thin airfoil in combination with a saturated gust loads alleviation system.

\section{Copyright Notice}

The author has retained copyright of the publication and releases it to the public according to the terms of the DLR elib archive.

\section{Citation Notice}

[1] Andreas Knoblach, Harald Pfifer, and Peter Seiler. Worst case analysis of a saturated gust loads alleviation system. In Guidance, Navigation, and Control Conference. AIAA, 2015. doi:10.2514/6.2015-0858.

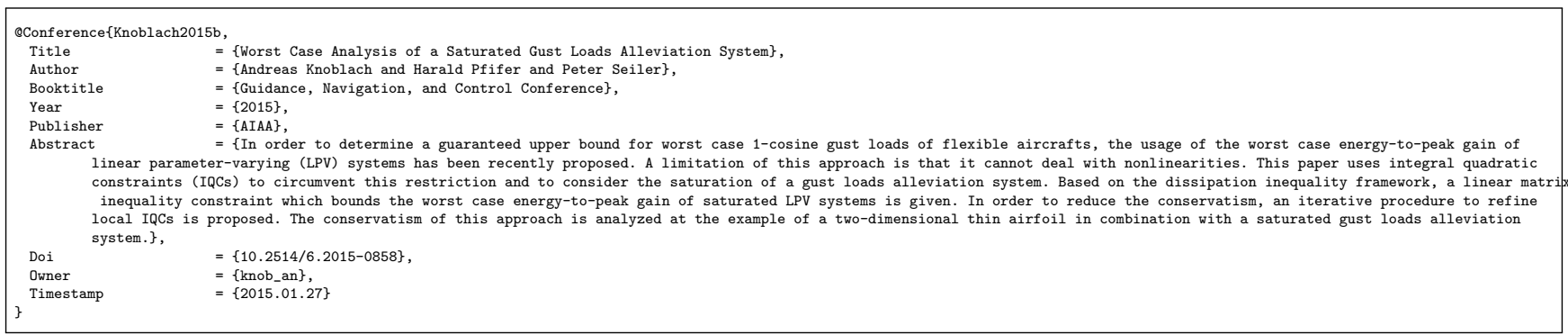




\title{
Worst Case Analysis of a Saturated Gust Loads Alleviation System
}

\author{
Andreas Knoblach* \\ Institute of System Dynamics and Control, German Aerospace Center (DLR), 82234 Weßling, Germany \\ Harald Pfifer ${ }^{\dagger}$ and Peter Seiler ${ }^{\ddagger}$ \\ Department of Aerospace Engineering 83 Mechanics, University of Minnesota, Minneapolis
}

\begin{abstract}
In order to determine a guaranteed upper bound for worst case "1-cosine" gust loads of flexible aircrafts, the usage of the worst case energy-to-peak gain of linear parametervarying (LPV) systems has been recently proposed. A limitation of this approach is that it cannot deal with nonlinearities. This paper uses integral quadratic constraints (IQCs) to circumvent this restriction and to consider the saturation of a gust loads alleviation system. Based on the dissipation inequality framework, a linear matrix inequality constraint which bounds the worst case energy-to-peak gain of saturated LPV systems is given. In order to reduce the conservatism, an iterative procedure to refine local IQCs is proposed. The conservatism of this approach is analyzed at the example of a two-dimensional thin airfoil in combination with a saturated gust loads alleviation system.
\end{abstract}

\section{Introduction}

In order to certify a new aircraft, it must be proved that it can withstand loads caused by turbulence and gusts. ${ }^{1}$ According to the CS-25 of the European Aviation Safety Agency (EASA), ${ }^{2}$ two types of excitations have to be considered: discrete "1-cosine" gusts and continuous turbulence. In the first case, an aircraft model is excited with a single "1-cosine" gust profile and the model outputs, e. g. the wing root bending moment (WRB) or the vertical accelerations, are computed and the simulation is repeated for several gust lengths. Moreover, the complete process has to be performed for many flight points such that the entire flight envelope (defined by velocity, altitude, loading etc.) is covered. Finally, the maximum and minimum peak of every output for all these simulations define the limit loads due to discrete gusts. The other type of excitation - namely continuous turbulence - considers the stochastic nature of turbulence. Here, the von Karman wind turbulence model is used to excite the aircraft and the results are evaluated by stochastic means. The limit loads are derived from the root mean square (RMS) value of the model response. A detailed description of discrete gusts and continuous turbulence scenarios can be found in Ref. 1.

Since these millions of simulations are very time consuming, there is a need for fast and reliable algorithms to identify worst case flight points and to compute maximum loads. To that end, it has been recently proposed in Ref. 3 to use the worst case energy-to-peak gain or mathematically speaking the induced $\mathcal{L}_{2} \rightarrow \mathcal{L}_{\infty}$ norm. This approach allows an extremely fast computation of an upper bound for the peak loads. However, this method is restricted to parametrically uncertain or linear parameter-varying (LPV) systems. Since a nonlinear gust load alleviation system has to be considered in many cases, the applicability of using the worst case energy-to-peak gain is limited.

The major nonlinearity of gust load alleviation systems is saturation. Since the interconnections of linear time invariant (LTI) systems and saturation occur very often in practice, they are intensively studied in the literature. Most approaches can be put into the context of integral quadratic constraints (IQCs), see e.g. Refs. 4, 5, and 6. Recent results in Ref. 7 extend the IQC framework to LPV systems, which allows the analysis of saturated LPV systems.

\footnotetext{
* Research Associate, andreas.knoblach@dlr.de.

$\dagger$ Postdoctoral Associate, hpfifer@umn.edu.

$\ddagger$ Assistant Professor, seiler@aem.umn. edu.
} 
The aim of this paper is to investigate how IQCs can be used for the analysis of a saturated gust loads alleviation system. The paper is structured as follows: The interconnection of an LPV system and saturation is described in Section II. A brief summary on IQCs is provided in Section III. The focus lies on the description of saturation by IQCs. The analysis interconnection and an upper bound for the worst case energy-to-peak gain of saturated LPV systems is given in Section IV. Additionally, an iterative procedure to refine local IQCs is proposed. The effectiveness of the proposed method is investigated in Section V. This investigation is pursued at the example of a two-dimensional thin airfoil in combination with a gust load alleviation system.

\section{Saturated LPV Systems}

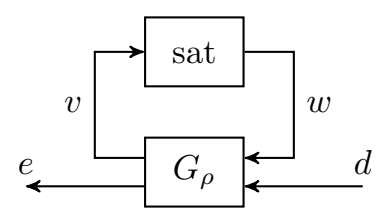

(a) Feedback interconnection of an LPV system and saturation

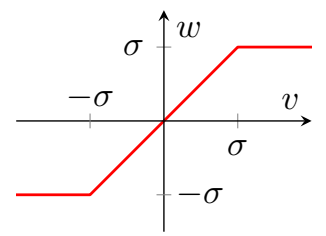

(b) Saturation nonlinearity

Figure 1. Saturated LPV system

The paper considers a system under saturation given by the feedback interconnection, denoted $\mathcal{F}_{u}\left(G_{\rho}\right.$, sat), of an LPV plant $G_{\rho}$ and a saturation nonlinearity sat as shown in Figure 1(a). The saturation $w=\operatorname{sat}(v)$ is defined by

$$
w=\operatorname{sat}(v)= \begin{cases}v & \text { if }|v|<\sigma \\ \operatorname{sgn}(v) \cdot \sigma & \text { otherwise, }\end{cases}
$$

where $\sigma$ denotes the saturation limit. The saturation nonlinearity is depicted in Figure 1(b). LPV systems are a class of linear systems whose state space matrices dependent on a scheduling parameter vector $\rho: \mathbb{R} \rightarrow \mathbb{R}^{n_{\rho}}$. The parameter is assumed to be a continuously differentiable function of time and the admissible trajectories are restricted based on physical considerations to a compact subset $\mathcal{P} \subset \mathbb{R}^{n_{\rho}}$. In addition, the parameter rates of variation $\dot{\rho}$ are assumed to lie within the hyperrectangle $\dot{\mathcal{P}}$ defined by

$$
\dot{\mathcal{P}}:=\left\{q|| q_{i} \mid \leq \nu_{i} \forall i \in\left\{1, \ldots, n_{\rho}\right\}\right\},
$$

where $\nu_{i}$ are nonnegative numbers. The set of all admissible trajectories is defined as

$$
\mathcal{A}:=\{\rho \mid \rho(t) \in \mathcal{P}, \dot{\rho}(t) \in \dot{\mathcal{P}} \forall t \geq 0\} .
$$

The state-space matrices of an LPV system are continuous functions of the parameter, e.g. $A: \mathcal{P} \rightarrow \mathbb{R}^{n_{x} \times n_{x}}$. An $n_{x}^{\text {th }}$ order LPV system $G_{\rho}$ is defined by

$$
\begin{aligned}
\dot{x}(t) & =A(\rho(t)) x(t)+B(\rho(t))\left[\begin{array}{l}
w(t) \\
d(t)
\end{array}\right] \\
{\left[\begin{array}{l}
v(t) \\
e(t)
\end{array}\right] } & =C(\rho(t)) x(t)+D(\rho(t))\left[\begin{array}{l}
w(t) \\
d(t)
\end{array}\right] .
\end{aligned}
$$

Throughout the remainder of the paper, the explicit dependence on $t$ is occasionally suppressed to shorten the notation.

\section{Integral Quadratic Constraints for Saturation}

This section describes constraints on the saturation function that can be incorporated in the input/output analysis. The constraints are taken from Ref. 4 and fit in the more general framework of IQCs, which bound the input/output signals of a bounded causal operator $w=\Delta(v)$. These constraints can be expressed in the frequency domain as well as in the time domain. First, a definition for an IQC in the frequency domain is given. 
Definition 1: Let $\Pi: \mathrm{jR} \rightarrow \mathbb{C}^{\left(n_{v}+n_{w}\right) \times\left(n_{v}+n_{w}\right)}$ be a measurable Hermitian-valued function. A bounded, causal operator $\Delta: \mathcal{L}_{2 e}^{n_{v}} \rightarrow \mathcal{L}_{2 e}^{n_{w}}$ satisfies an IQC defined by $\Pi$ if the following inequality holds for all $v \in \mathcal{L}_{2}^{n_{v}}$, $w=\Delta(v)$, and $T \geq 0$

$$
\int_{-\infty}^{\infty}\left[\begin{array}{l}
\hat{v}(\mathrm{j} \omega) \\
\hat{w}(\mathrm{j} \omega)
\end{array}\right]^{*} \Pi(\mathrm{j} \omega)\left[\begin{array}{c}
\hat{v}(\mathrm{j} \omega) \\
\hat{w}(\mathrm{j} \omega)
\end{array}\right] \mathrm{d} \omega \geq 0 .
$$

In (6), $\hat{v}$ and $\hat{w}$ are Fourier transforms of $v$ and $w$, respectively.

The multiplier $\Pi$ can be factorized as

$$
\Pi(\mathrm{j} \omega)=\Psi(\mathrm{j} \omega)^{*} M \Psi(\mathrm{j} \omega)
$$

which allows to connect the frequency domain formulation to a time-domain formulation. ${ }^{4,8,9}$ This leads to Definition 2 for an IQC in the time domain. It should be noted that there are some technical restrictions in the factorization of IQCs, see Refs. 4, 8, and 10. However, these restrictions do not apply to the IQCs considered in this paper.

Definition 2: Let $M$ be a symmetric matrix, i.e. $M=M^{\mathrm{T}} \in \mathbb{R}^{n_{z} \times n_{z}}$, and $\Psi$ a stable linear system, i.e. $\Psi \in \mathbb{R H}_{\infty}^{n_{z} \times\left(n_{v}+n_{w}\right)}$. A bounded, causal operator $\Delta: \mathcal{L}_{2 e}^{n_{v}} \rightarrow \mathcal{L}_{2 e}^{n_{w}}$ satisfies an IQC defined by $(\Psi, M)$ if the following inequality holds for all $v \in \mathcal{L}_{2}^{n_{v}}, w=\Delta(v)$, and $T \geq 0$

$$
\int_{0}^{T} z(t)^{\mathrm{T}} M z(t) \mathrm{d} t \geq 0
$$

where $z$ is the output of the linear system $\Psi$

$$
\begin{aligned}
\dot{x}_{\Psi} & =A_{\Psi} x_{\Psi}+B_{\Psi 1} v+B_{\Psi 2} w, \quad x_{\Psi}(0)=0 \\
z & =C_{\Psi} x_{\Psi}+D_{\Psi 1} v+D_{\Psi 2} w .
\end{aligned}
$$

The notation $\Delta \in \operatorname{IQC}(\Psi, M)$ is used if $\Delta$ satisfies the IQC defined by $(\Psi, M)$.

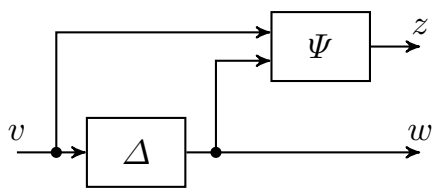

Figure 2. Graphical interpretation of the IQC

Figure 2 provides a graphical interpretation of a time domain IQC. The input and output signals of $\Delta$ are filtered through $\Psi$. If $\Delta \in \operatorname{IQC}(\Psi, M)$, then the output signal $z$ satisfies the (time-domain) constraint in (8) for any finite-horizon $T \geq 0$. Three classical IQCs from Ref. 4 for saturation are given in the following subsections.

\section{III.A. Memoryless Sector Bounded Nonlinearity}

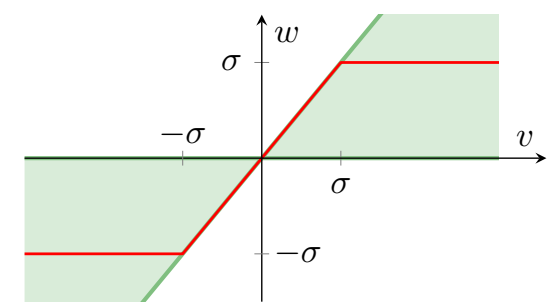

Figure 3. Sector constraint ( $\square$ ) for the saturation nonlinearity ( $\square$ )

It is easy to see in Figure 3 that saturation lies in the sector defined by two lines with slope $\alpha=0$ and $\beta=1$, denoted as sector $[0,1]$. More general, a memoryless nonlinearity $\Phi: \mathbb{R} \rightarrow \mathbb{R}$ lies in sector $[\alpha, \beta]$ if

$$
(w(t)-\alpha v(t))(\beta v(t)-w(t)) \geq 0
$$


holds for all $v(t) \in \mathbb{R}$ and $w(t)=\phi(v(t))$. This can be equivalently expressed as

$$
\left[\begin{array}{c}
v(t) \\
w(t)
\end{array}\right]^{\mathrm{T}}\left[\begin{array}{cc}
-2 \alpha \beta & \alpha+\beta \\
\alpha+\beta & -2
\end{array}\right]\left[\begin{array}{l}
v(t) \\
w(t)
\end{array}\right] \geq 0 .
$$

Since (11) has to hold pointwise in time, it also holds when integrated over time

$$
\int_{0}^{T}\left[\begin{array}{c}
v(t) \\
w(t)
\end{array}\right]^{T}\left[\begin{array}{cc}
-2 \alpha \beta & \alpha+\beta \\
\alpha+\beta & -2
\end{array}\right]\left[\begin{array}{c}
v(t) \\
w(t)
\end{array}\right] \mathrm{d} t \geq 0
$$

for any $T \geq 0$. Note that the converse is not true. Hence, the saturation function satisfies the IQC defined by

$$
\Psi=\mathrm{I}_{2} \quad \text { and } \quad M=\left[\begin{array}{cc}
-2 \alpha \beta & \alpha+\beta \\
\alpha+\beta & -2
\end{array}\right],
$$

with $\alpha=0$ and $\beta=1$. Note that the sector IQC holds also for time varying, memoryless nonlinearities $w(t)=\phi(v(t), t)$.

\section{III.B. Popov IQC}

In order to capture the time invariance of saturation, the Popov IQC can be used. Consider a memoryless and time invariant nonlinearity $w(t)=\phi(v(t))$ where $\phi: \mathbb{R} \rightarrow \mathbb{R}$ is a continuous function with $v(0)=0$, and $w$ and $\dot{v}$ are square integrable. Then, $v$ and $w$ satisfy the IQC defined by

$$
\Pi(\mathrm{j} \omega)= \pm\left[\begin{array}{cc}
0 & \mathrm{j} \omega \\
-\mathrm{j} \omega & 0
\end{array}\right]
$$

However, since $\Pi$ is not bounded on the imaginary axis, it is not a proper IQC. As a remedy, the loop transformation from Figure 4 can be used. The operator $\bar{\Delta}$ satisfies the IQC defined by

$$
\bar{\Pi}(\mathrm{j} \omega)= \pm\left[\begin{array}{cc}
0 & \frac{\mathrm{j} \omega}{1-\mathrm{j} \omega} \\
\frac{-\mathrm{j} \omega}{1+\mathrm{j} \omega} & 0
\end{array}\right]
$$

In order to revert the low-pass filter, the non-proper filter $s+1$ must be added to the interconnection (see Figure 4). This requires that the plant is strictly proper. The combination of the Popov IQC and of the sector constraint IQC yields the classical Popov criterion. ${ }^{4}$

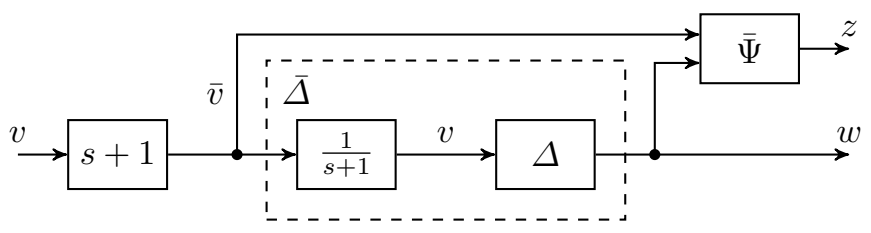

Figure 4. Popov IQC: loop transformation to yield a proper IQC

\section{III.C. Zames-Falb IQC}

Finally, saturation is described by a monotonic, slope restricted, and odd function which is the last property satisfying a classical IQC. Consider $w(t)=\phi(v(t))$ where $\phi: \mathbb{R} \rightarrow \mathbb{R}$ is a monotonic and odd function and its slope is bounded by $\frac{\partial \phi}{\partial v} \in[0, k]$ for a constant $k>0$. Then $v$ and $w$ satisfy the IQC

$$
\Pi(\mathrm{j} \omega)=\left[\begin{array}{cc}
0 & 1+H(\mathrm{j} \omega) \\
1+H(\mathrm{j} \omega)^{*} & -\left(2+H(\mathrm{j} \omega)+H(\mathrm{j} \omega)^{*}\right) / k
\end{array}\right]
$$

where $H(s)$ is an arbitrary rational transfer function whose impulse response has an $\mathcal{L}_{1}$ norm not larger than one. Details can be found in Ref. 11. 


\section{Robust Performance Analysis of Saturated LPV Systems}

\section{IV.A. Uncertain LPV Systems}

In Ref. 7, it is proposed to describe an uncertain LPV system by the feedback interconnection of an LPV system $G_{\rho}$ with an nonlinear and/or uncertain operator $\Delta$. This corresponds to an upper linear fractional transformation (LFT), which is denoted $\mathcal{F}_{u}\left(G_{\rho}, \Delta\right)$. The exact relation $w=\Delta(v)$ is replaced by an IQC such that $\Delta \in \operatorname{IQC}(\Psi, M)$. The dynamics of the resulting analysis interconnection (see Figure 5 ) is ruled by

$$
\begin{aligned}
\dot{x} & =A(\rho) x+B_{1}(\rho) w+B_{2}(\rho) d \\
z & =C_{1}(\rho) x+D_{11}(\rho) w+D_{12}(\rho) d \\
e & =C_{2}(\rho) x+D_{21}(\rho) w+D_{22}(\rho) d .
\end{aligned}
$$

Note that $x$ includes the states of $G_{\rho}$ and $\Psi$. In the following analysis, the uncertainty $\Delta$ is removed and $w$ is treated as an external signal constrained by (8).

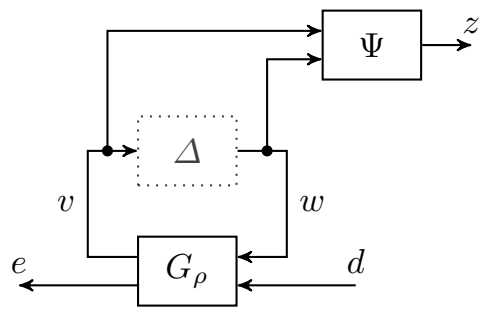

Figure 5. Analysis interconnection

\section{IV.B. Energy-to-Peak Gain of Uncertain LPV Systems}

Although the induced $\mathcal{L}_{2} \rightarrow \mathcal{L}_{2}$ norm (or for LTI system also $\mathcal{H}_{\infty}$ norm) is commonly used in the robust performance analysis framework, the induced $\mathcal{L}_{2} \rightarrow \mathcal{L}_{\infty}$ is more suited for loads analysis since it allows to compute an upper bound for the output peaks. The induced $\mathcal{L}_{2} \rightarrow \mathcal{L}_{\infty}$ norm of an uncertain LPV system is defined as

$$
\left\|\mathcal{F}_{u}\left(G_{\rho}, \Delta\right)\right\|_{\mathcal{L}_{2} \rightarrow \mathcal{L}_{\infty}}:=\sup _{\Delta \in \operatorname{IQC}(\Psi, M)} \sup _{\substack{d \in \mathcal{L}_{2} \backslash\{0\} \\ \rho \in \mathcal{A}}} \frac{\|e\|_{\mathcal{L}_{\infty}}}{\|d\|_{\mathcal{L}_{2}}} .
$$

In (18),

$$
\|x\|_{\mathcal{L}_{2}}=\int_{0}^{\infty}\left(x(t)^{\mathrm{T}} x(t)\right)^{\frac{1}{2}} \mathrm{~d} t \quad \text { and } \quad\|x\|_{\mathcal{L}_{\infty}}=\sup _{t}\left(x(t)^{\mathrm{T}} x(t)\right)^{\frac{1}{2}}
$$

represent the $\mathcal{L}_{2}$ (or energy) signal norm and the $\mathcal{L}_{\infty}$ (or peak) signal norm, respectively. The notations $x \in \mathcal{L}_{2}$ and $x \in \mathcal{L}_{\infty}$ are used to refer to signals with finite $\mathcal{L}_{2}$ and $\mathcal{L}_{\infty}$ norm. The induced $\mathcal{L}_{2} \rightarrow \mathcal{L}_{\infty}$ norm represents hence the worst case energy-to-peak gain over all uncertainties that satisfy the IQC $(\Psi, M)$ and over all admissible parameter trajectories.

Using the interconnection in (17), a theorem based on the dissipation inequality framework can be formulated which bounds the induced $\mathcal{L}_{2} \rightarrow \mathcal{L}_{\infty}$ norm. The analysis leads to conditions that include the parameter and the parameter rate at a single point in time, i. e. $(\rho(t), \dot{\rho}(t))$. In order to emphasize that the conditions depend only on the sets $\mathcal{P}$ and $\dot{\mathcal{P}}$, the parametric notation $(p, q) \in \mathcal{P} \times \dot{\mathcal{P}}$ is introduced. The following theorem is a minor modification of the worst case condition for the induced $\mathcal{L}_{2} \rightarrow \mathcal{L}_{2}$ norm given in Ref. 7.

Theorem 1: The induced $\mathcal{L}_{2} \rightarrow \mathcal{L}_{\infty}$ norm from $d$ to e of an uncertain LPV system $\left\|\mathcal{F}_{u}\left(G_{\rho}, \Delta\right)\right\|_{\mathcal{L}_{2} \rightarrow \mathcal{L}_{\infty}}$ with $D_{21}(\rho)=0$ and $D_{22}(\rho)=0$ is smaller than a performance index $\gamma$ if the interconnection is well posed for all $\Delta \in \operatorname{IQC}(\Psi, M)$ and if there exists a continuous differentiable Lyapunov matrix function $X: \mathcal{P} \rightarrow \mathbb{R}^{n_{x} \times n_{x}}$ with $X^{\mathrm{T}}=X$ and a scalar $\lambda>0$ s.t. for all $(p, q) \in \mathcal{P} \times \dot{\mathcal{P}}$

$$
X(p)>0,
$$




$$
\begin{gathered}
{\left[\begin{array}{ccc}
A(p)^{\mathrm{T}} X(p)+X(p) A(p)+\sum_{i=1}^{n_{\rho}} \frac{\partial X(p)}{\partial p_{i}} q_{i} & X(p) B_{1}(p) & X(p) B_{2}(p) \\
B_{1}(p)^{\mathrm{T}} X(p) & 0 & 0 \\
B_{2}(p)^{\mathrm{T}} X(p) & 0 & -\gamma \mathrm{I}
\end{array}\right]} \\
+\lambda\left[\begin{array}{c}
C_{1}(p)^{\mathrm{T}} \\
D_{11}(p)^{\mathrm{T}} \\
D_{12}(p)^{\mathrm{T}}
\end{array}\right] M\left[\begin{array}{lll}
C_{1}(p) & D_{11}(p) & D_{12}(p)
\end{array}\right]<0, \\
{\left[\begin{array}{cc}
X(p) & C_{2}(p)^{\mathrm{T}} \\
C_{2}(p) & \gamma \mathrm{I}
\end{array}\right]>0 .}
\end{gathered}
$$

Proof: The proof is based on defining a storage function $V: \mathbb{R}^{n_{x}} \times \mathcal{P} \rightarrow \mathbb{R}^{+}$

$$
V(x, \rho):=x^{\mathrm{T}} X(\rho) x .
$$

Left and right multiply (20b) by $\left[\begin{array}{lll}x^{T} & w^{T} & d^{T}\end{array}\right]$ and its transpose, respectively, to show that $V$ satisfies the dissipation inequality

$$
\dot{V}(t)+\lambda z(t)^{\mathrm{T}} M z(t) \leq \gamma d(t)^{\mathrm{T}} d(t) .
$$

The dissipation inequality can be integrated from $t=0$ to $t=T$ with the initial condition $x(0)=0$. Along with the IQC condition (8) and $\lambda>0$ this leads to

$$
V(T) \leq \gamma \int_{0}^{T} d(t)^{\mathrm{T}} d(t) \mathrm{d} t .
$$

A Schur complement on (20c) and the left and right multiplication with $x^{\mathrm{T}}$ and $x$ yield

$$
\frac{1}{\gamma} e(T)^{\mathrm{T}} e(T) \leq V(T)
$$

Combine (23) and (24) and take the supremum w.r.t. $T$ to conclude

$$
\|e\|_{\mathcal{L}_{\infty}} \leq \gamma\|d\|_{\mathcal{L}_{2}}
$$

A detailed proof of the analogous induced $\mathcal{L}_{2}$ norm can be found in Ref. 7 .

An upper bound for the worst case energy-to-peak gain can be obtained from Theorem 1 by solving a semidefinite program in the variables $X, \lambda$ and $\gamma$. In order to arrive at numerically tractable conditions, a gridding based approximation of the parameter space is used and basis functions are assigned for $X(p)$, see Refs. 12 and 13 for details. Note that for simplification the theorem is only given in terms of a single IQC $(\Psi, M)$. It can be easily extended to handle multiple IQCs. ${ }^{7}$

\section{IV.C. Analysis using Local IQCs}

In Section III, standard constraints from the literature ${ }^{4,11}$ for the saturation function have been given. While all these IQCs guarantee global robust performance of a system under saturation, all of them are independent of the saturation level. Hence, they are expected to be very conservative for many practical applications. It is well understood in the literature that performance results that only hold locally can greatly improve the analysis of saturated systems by incorporating the actual saturation level, see Refs. 14, 15, and 16. If the global performance requirement is relaxed to only consider local performance, a modified, less conservative version of the sector bound IQC can be used. The approach pursued in this paper is based on the notion of local IQCs introduced in Ref. 17. There, a general framework for the local analysis of systems under perturbations described by IQCs is given. In the present paper, the specific nature of the perturbation, namely the saturation function, is exploited to obtain local conditions. The conditions are similar to the IQCs for saturations given in Ref. 5 .

The global sector constraint for the saturation function is defined by the sector $[0,1]$. It is easily seen that the sector bound holds independent of the size of the input $v$, see Figure 6 . If an $\mathcal{L}_{\infty}$ norm bound on the input $v$ is known, i.e. $|v| \leq R$, then a refined sector constraint can be given. Instead of checking over the 


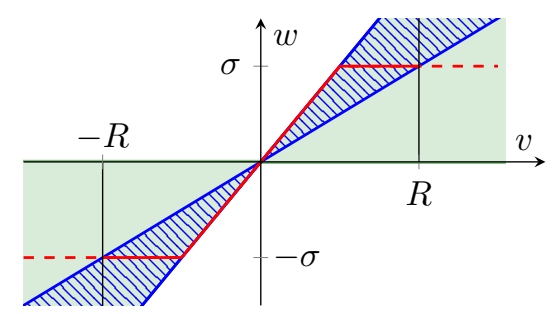

Figure 6. Local sector constraint IQC for saturation: Because of $|v| \leq R$, only the solid drawn part of the saturation ( can be reached, but not the dashed one (- - ). This allows to decrease the sector from ( $\square)$ to (MU).

whole sector $[0,1]$, it is sufficient to only consider the smaller sector $[\sigma / R, 1]$. Recall that $\sigma$ is the saturation level. The smaller, local sector is depicted in Figure 6. In order to compute a valid bound on $v$, a bounded disturbance energy $\|d\|_{\mathcal{L}_{2}} \leq 1$ is assumed. Then, the induced $\mathcal{L}_{2} \rightarrow \mathcal{L}_{\infty}$ norm from the performance input $d$ to the saturation input $v$ provides a valid bound on $v$.

In order to reduce the conservatism of the results, a possibly small bound on $v$ should be determined. This can be achieved by the iterative procedure in Algorithm 1.

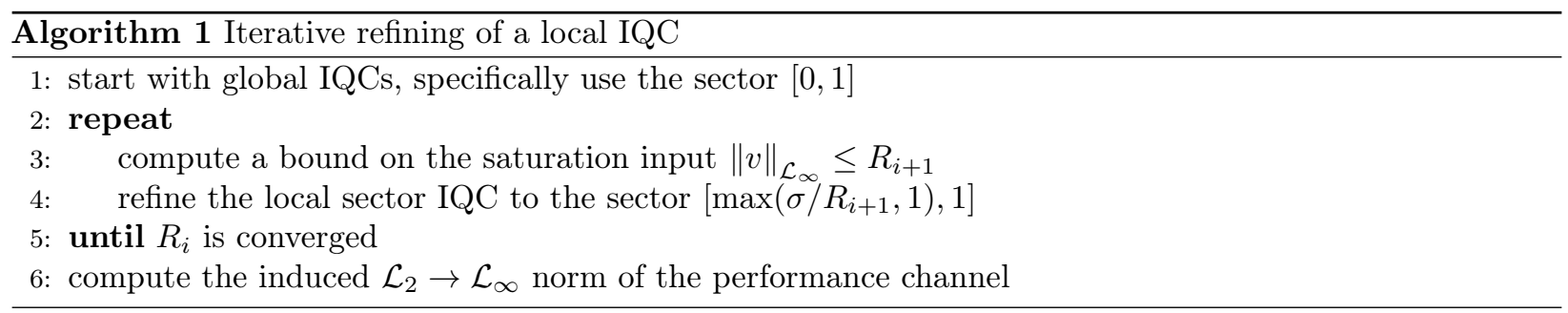

In Line 1, the saturation is described by the globally valid IQCs from Section III. Specifically, the sector $[0,1]$ is used. Line 3 consists in computing the induced $\mathcal{L}_{2} \rightarrow \mathcal{L}_{\infty}$ norm from $d$ to $v$ using Theorem 1 . In combination with $\|d\|_{\mathcal{L}_{2}} \leq 1$, this norm provides an $\mathcal{L}_{\infty}$ norm bound $R_{i}$ on $v$. This bound is used in Line 4 to define the locally valid sector constraint $\left[\max \left(\sigma / R_{i+1}, 1\right), 1\right]$. Lines 3 and 4 are repeated until $R_{i}$ is converged. In the last step (Line 6), an upper bound for the worst case energy-to-peak gain of the performance channel is computed. Note that in the last step the more common worst case energy gain can be alternatively considered using Theorem 2 in Ref. 7.

\section{Analysis of a Saturated Gust Loads Alleviation System}

The proposed approach is used to compute the worst case energy-to-peak gain of a simple aeroservoelastic system. A two degree of freedom airfoil in combination with a gust load alleviation system is considered. The effects of different IQCs, several saturation limits and parameter rate bounds are investigated.

\section{V.A. Aeroservoelastic System}

The considered aeroelastic system, taken from Ref. 18, is illustrated in Figure 7. The equations of motion are

$$
\left[\begin{array}{cc}
m & m \cdot x_{\mathrm{cg}} \\
m \cdot x_{\mathrm{cg}} & I
\end{array}\right]\left[\begin{array}{l}
\ddot{h} \\
\ddot{\alpha}
\end{array}\right]+\left[\begin{array}{cc}
k_{h} & 0 \\
0 & k_{\alpha}
\end{array}\right]\left[\begin{array}{l}
h \\
\alpha
\end{array}\right]=\left[\begin{array}{c}
-L \\
\tau
\end{array}\right]
$$

where $h$ denotes the vertical deflection and $\alpha$ the pitch angle. ${ }^{\text {a }}$ The lift $L$ and the pitching moment $\tau$ are given by

$$
\left[\begin{array}{c}
-L \\
\tau
\end{array}\right]=\frac{1}{2} \rho v^{2}\left[Q_{\text {eig }}(s) \mid Q_{\text {gust }}(s)\right]\left[\begin{array}{c}
h \\
\alpha \\
\beta \\
\hline v_{\text {gust }}
\end{array}\right] .
$$

The Theodorsen function $Q_{\text {eig }}(s)$ models the relation between the aerodynamic forces and the heave, the pitching and the flap movement, denoted $h, \alpha$, and $\beta$, respectively. The Sears function $Q_{\text {gust }}(s)$ maps the

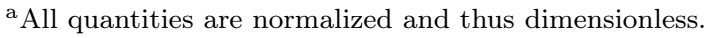




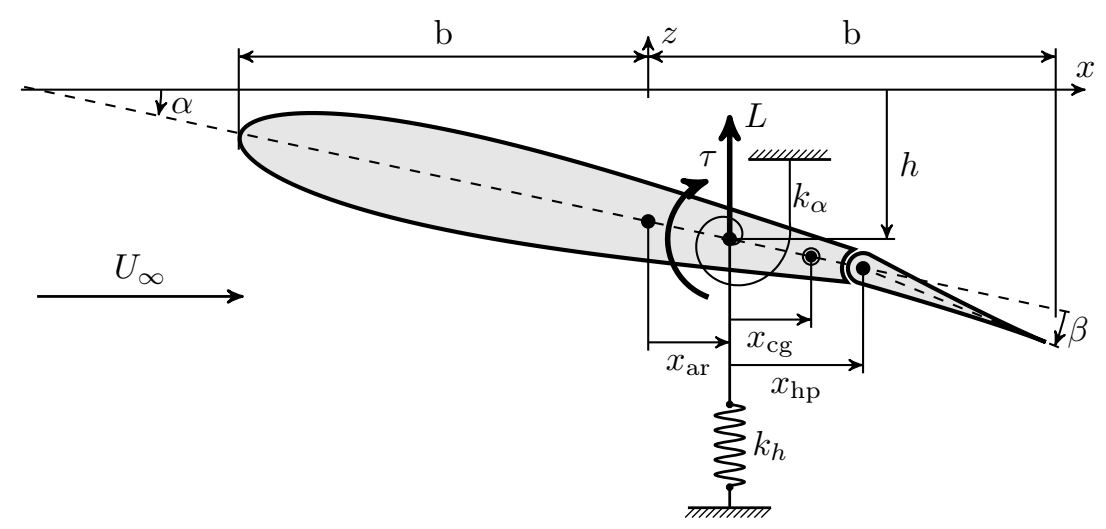

Figure 7. Aeroservoelastic system

vertical gust velocity $v_{\text {gust }}$ on $L$ and $\tau$. Both transfer functions are non-rational in the Laplace domain. In order to yield a state space realization, R. T. Jones' rational approximation is used. The functions depend on the free stream velocity $U_{\infty}$, the half chord length $b$, the location of the aerodynamic reference axis $x_{\mathrm{ar}}$, and the hinge position $x_{\mathrm{hp}} . Q_{\text {eig }}$ and $Q_{\text {gust }}$ are explicitly given in Ref. 18. In this paper, the velocity $U_{\infty}$ is considered as scheduling parameter. Ten equidistantly spaced grid points in the interval $U_{\infty} \in[0.3,0.9]$ are used. The rate bounds are $\dot{U}_{\infty} \in[-0.1,0.1]$. The other parameters are compiled in Table 1.

Table 1. Model parameters

\begin{tabular}{llc}
\hline Sym. & Explanation & Value \\
\hline$k_{h}$ & translational stiffness & 0.04 \\
$k_{\alpha}$ & rotational stiffness & 0.25 \\
$m$ & mass & 1.00 \\
$I$ & inertia & 0.25 \\
$x_{\mathrm{cg}}$ & location of the center of gravity & 0.20 \\
\hline$\rho$ & air density & 0.80 \\
$b$ & half chord length & 1.00 \\
$x_{\mathrm{ar}}$ & location of the aerodynamic reference axis & -0.20 \\
$x_{\mathrm{hp}}$ & location of the hinge axis & 0.80 \\
\hline
\end{tabular}

The close loop configuration in Figure 8 is considered. The weighting filter

$$
W_{\text {Gust }}:\left\{\begin{array}{c}
\dot{x}_{W}=\frac{1}{10} x_{W}+\frac{1}{10} d \\
v_{\text {gust }}=x_{W}
\end{array}\right.
$$

is used to shape suitable gusts with unit energy. ${ }^{3}$ The performance outputs are the spring forces

$$
e=\left[\begin{array}{l}
e_{h} \\
e_{\alpha}
\end{array}\right]=\left[\begin{array}{l}
k_{h} \cdot h \\
k_{\alpha} \cdot \alpha
\end{array}\right] .
$$

The first oder low pass filter

$$
G_{\mathrm{act}}:\left\{\begin{aligned}
\dot{x}_{\mathrm{act}} & =x_{\mathrm{act}}+u \\
v & =x_{\mathrm{act}}
\end{aligned}\right.
$$

serves as an actuator model. Since the flap deflection $\beta$ is assumed to be limited, saturation

$$
\beta=w=\operatorname{sat}(v)
$$


is added. A simple gust load alleviation system which is based on a gain scheduled (by $U_{\infty}$ ) proportional controller

$$
K:\left\{u=\left(\left[\begin{array}{lll}
4.2 & -27.3 & 149.2
\end{array}\right]+U_{\infty}\left[\begin{array}{lll}
0.19 & -49.4 & 138.6
\end{array}\right]\right)\left[\begin{array}{c}
v_{\text {gust }} \\
e_{\alpha} \\
e_{\alpha}
\end{array}\right]\right.
$$

is used to close the loop. The controller is tuned using systune. ${ }^{19}$ The tuning goal is to minimize the variance of the performance output.

Since the parameter dependence of the resulting state space model is highly involved, it is not explicitly given. However, it has ten states. A Bode diagram of the open and the nominal closed loop (i. e. without saturation) is depicted in Figure 9 for different parameter values.

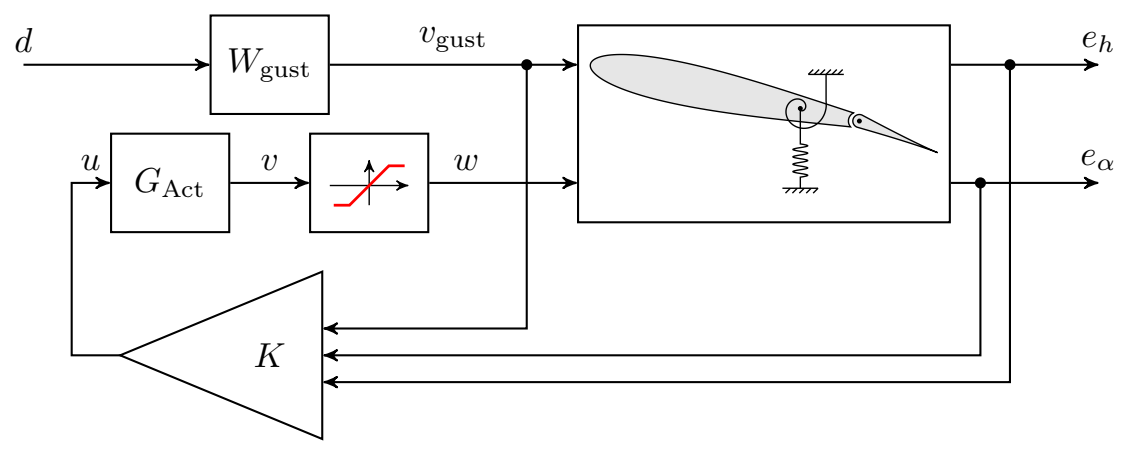

Figure 8. Closed loop of the aeroservoelastic system
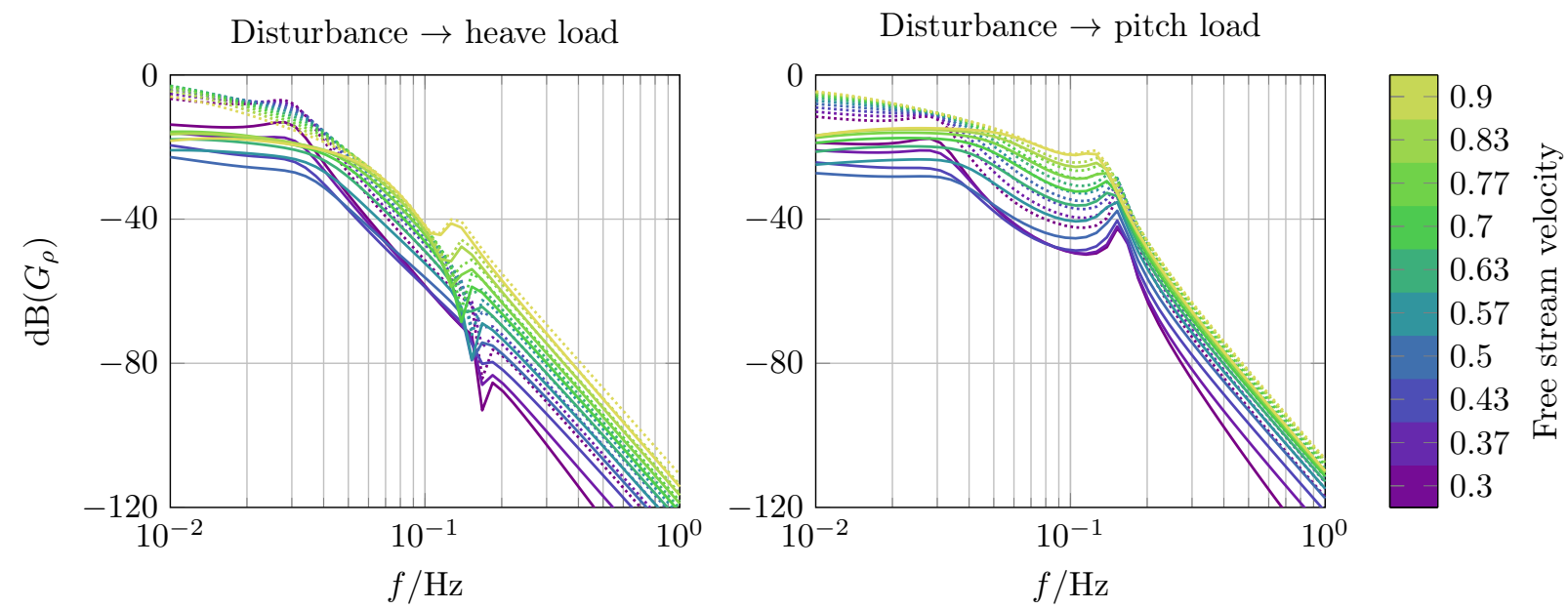

Figure 9. Bode diagram of the aeroservoelastic system: (…….) represents the open loop and ( $\longrightarrow$ ) the nominal closed loop. The color indicates the free stream velocity.

\section{V.B. Comparison of IQCs}

The effect of different IQCs is investigated using the LTI model corresponding to the highest free stream velocity. The globally valid sector $[0,1]$ is considered separately, in combination with the Popov IQC and in combination with three different parameterizations of the Zames-Falb IQC. The Zames-Falb IQC is parameterized by a first order low pass filter

$$
H(s)=\frac{1}{\frac{1}{T_{\mathrm{ZF}}} s+1},
$$

where the three values for the time constant $T_{\mathrm{ZF}} \in\{0.01,0.1,1\}$ are used. Finally, the combination of the sector IQC, the Popov IQC, and all three Zames-Falb IQCs is considered. The results are compiled in Table 2. The best result is achieved using the sector IQC and the Zames-Falb IQC with $T_{\mathrm{ZF}}=0.1$. The results cannot be improved by adding the Popov IQC or combining several Zames-Falb IQCs. For that reason, the sector and the Zames-Falb IQCs with $T_{\mathrm{ZF}}=0.1$ are used below. 
Table 2. Results for several IQC combinations

\begin{tabular}{|c|c|c|c|c|c|c|}
\hline$T_{\mathrm{ZF}}$ & $\mathrm{Sec}$ & Sec/Pop & $\begin{array}{c}\mathbf{S e c} / \mathbf{Z F} \\
0.01\end{array}$ & $\begin{array}{c}\mathbf{S e c} / \mathbf{Z F} \\
0.1\end{array}$ & $\begin{array}{c}\mathbf{S e c} / \mathbf{Z F} \\
1\end{array}$ & $\begin{array}{c}\text { Sec } / \mathbf{P o p} / \mathbf{Z F} \\
\text { all }\end{array}$ \\
\hline Norm & 0.185 & 0.184 & 0.183 & 0.175 & 0.181 & 0.175 \\
\hline
\end{tabular}

\section{V.C. Convergence of Local IQCs}

The convergence behavior of Algorithm 1 is investigated in this subsection. The LPV model from Section V.A is used and the six saturation limits $\sigma \in\{0,3, \ldots 15\}$ are considered. The Lyapunov function is parameterized by a quadratic function. The results are depicted in Figure 10, where the induced $\mathcal{L}_{2} \rightarrow \mathcal{L}_{\infty}$ norm is plotted as a function of the iteration. In the left subplot, the norm from the disturbance to saturation input can be seen and in the right one, the norm of the performance channel.

First of all, a fast convergence after the third iteration can be recognized. The upper bound for the worst case energy-to-peak gain from the gust to the saturation input can be reduced by only $2 \%$. However, the proposed algorithm allows to reduce the upper bound for the performance channel by up to $69 \%$ (depending on the saturation limit).

In order to estimate the conservatism induced by the description of saturation with IQCs, the robust analysis results are compared to standard LPV analysis results for the open and the nominal closed loop. A saturation limit of zero corresponds with the open loop. Using Algorithm 1 for a zero saturation limit leads to conservative results, as can be seen in Figure 10 (compare (-๑) and (-)). The reason for this is that a zero saturation limit leads always to the sector $[0,1]$.

On the contrary, a very high saturation limit corresponds to the nominal closed loop. Specifically, an $\mathcal{L}_{\infty}$ norm of the saturation input less than the saturation limit results in a never saturated system. Consequently, the sector converges to $[1,1]$ which represents the smallest possible sector. In the considered example, the upper bound for the robust norm for a saturation limit of $\sigma=15(-\bullet)$ becomes even smaller than the result for the nominal closed loop $(-)$. The reason for this unexpected result is that the nominal LPV analysis is already conservative. In case of the robust analysis, the additional state of the Zames-Falb IQC introduces additional decision variables which reduce the conservatism. However, using a third order test function for the Lyapunov matrix function (

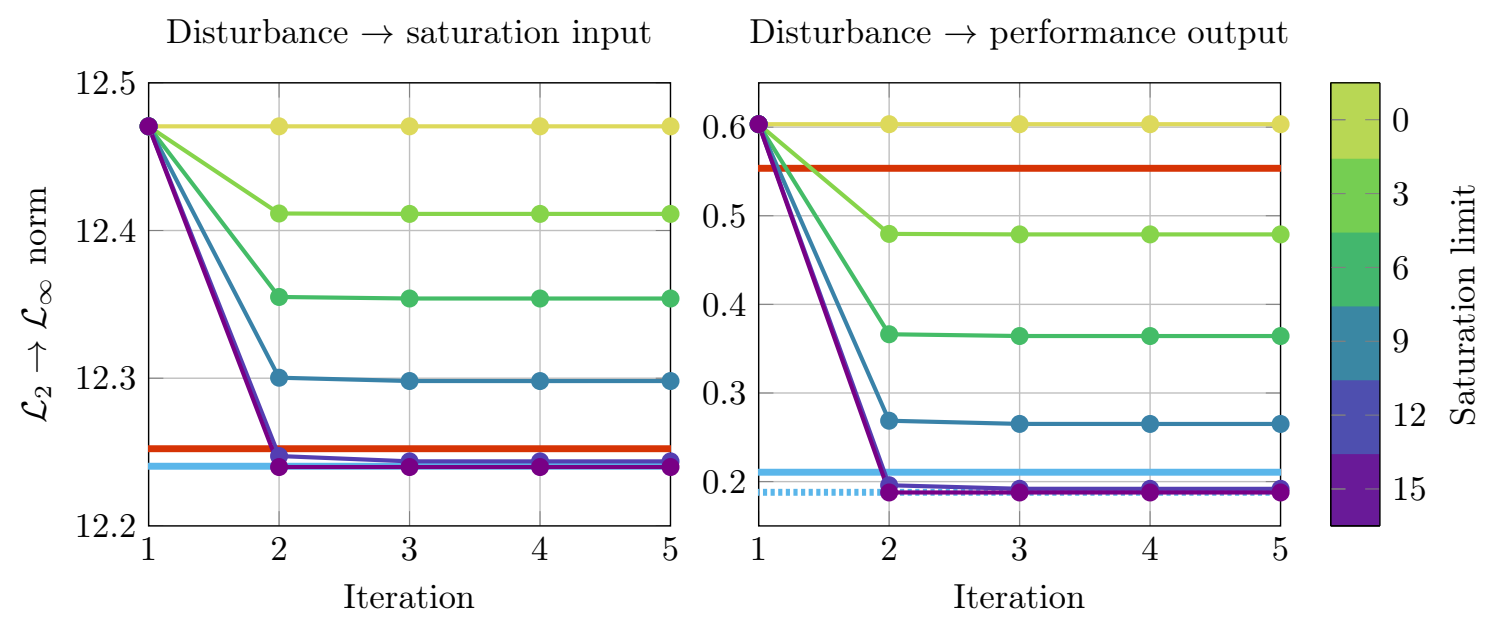

Figure 10. Convergence of local IQCs: The induced $\mathcal{L}_{2} \rightarrow \mathcal{L}_{\infty}$ gain is depicted as function of the iteration number ( $\longrightarrow$-) for several saturation limits indicated by the color. The results are compared to the open ( $\square$ ) and the nominal closed ( $\longrightarrow$ ) loop results. In case of the closed loop, a cubic test function for the Lyapunov matrix (

\section{V.D. Influence of the Parameter Rate Bounds}

In a last example, the influence of the parameter rate bounds is analyzed. To that end, Algorithm 1 is used to compute an upper bound of the induced $\mathcal{L}_{2} \rightarrow \mathcal{L}_{\infty}$ norm for the saturation limits $\sigma \in\{0,3, \ldots, 15\}$ and for the rate bounds $\dot{U}_{\infty} \in[-0.1 \nu, 0.1 \nu]$ with $\nu \in\{0.0,0.2, \ldots, 1.0\}$. The results are illustrated in Figure 11. 
Regarding the saturation limits, the results from the preceding subsection hold for all parameter rate bounds. For high saturation limits, the norm bounds converge to the nominal LPV analysis results ( - ). As expected, reducing the parameter rate bounds leads to a smaller upper bound. If the rate bound approaches zero, the robust analysis converges to the maximum LTI norm w. r.t. all ten nominal closed loop models $(\Delta)$. The latter norm is known to be not conservative and provides consequently a lower bound for the worst case performance.

Disturbance $\rightarrow$ performance output

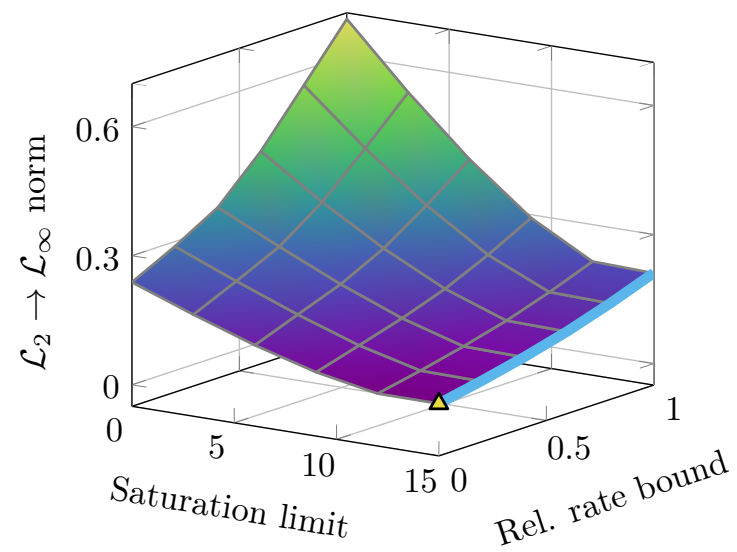

Figure 11. Influence of the parameter rate bounds: The induced $\mathcal{L}_{2} \rightarrow \mathcal{L}_{\infty}$ norm is computed for several rate bounds and saturation limits and compared to norms of the nominal closed loop ( $)$. Finally, $(\Delta)$ indicates the maximum LTI norm of the nominal closed loop.

Summarizing it can be said that the results are conservative if a low saturation limit is considered and the system is most of the time saturated. On the contrary, for relatively high saturation limits, Algorithm 1 converges to the nominal case and is hence only slightly conservative.

\section{Conclusions}

The analysis of saturated LPV systems using IQCs was discussed in this paper. In order to reduce the conservatism of the analysis results, an iterative procedure to refine local IQCs was proposed. This method was intensively studied at the example of a two-dimensional thin airfoil in combination with a gust load alleviation system. While this approach works well in case of relatively high saturation limits, the results are supposed to be conservative if the system is most of the time in saturation. However, this approach allows to compute guaranteed upper bounds for gust loads of flexible aircraft with a nonlinear gust load alleviation system. The meaningfulness of the nominal worst case energy-to-peak gain has been already demonstrated in Ref. 3. Whether the conservatism in terms of gust loads analysis is still acceptable or is too large for meaningful conclusions, has to be assessed using a generic aircraft model. This will be done in future work. Another point is to include further IQCs from Refs. 5 and 6.

\section{References}

${ }^{1}$ Hoblit, F. M., Gust loads on aircraft: concepts and applications, AIAA, 1988.

2EASA, Certification Specifications and Acceptable Means of Compliance for Large Aeroplanes CS-25, 2013.

${ }^{3}$ Knoblach, A., "Robust Performance Analysis Applied to Gust Loads Computation," Journal of Aeroelasticity and Structural Dynamics, Vol. 3, No. 1, 2013, pp. $39-50$.

${ }^{4}$ Megretski, A. and Rantzer, A., "System Analysis via Integral Quadratic Constraints," IEEE Transactions on Automatic Control, Vol. 42, No. 6, 1997, pp. $819-830$.

${ }^{5}$ Fang, H., Lin, Z., and Rotea, M., "On IQC approach to the analysis and design of linear systems subject to actuator saturation," System \& Control Letters, Vol. 57, 2008, pp. 611 - 619.

${ }^{6}$ Materassi, D. and Salapaka, M. V., "Less conservative absolute stability criteria using Integral Quadratic Constraints," American Control Conference, IEEE, 2009.

${ }^{7}$ Pfifer, H. and Seiler, P., "Robustness Analysis of Linear Parameter Varying Systems Using Integral Quadratic Constraints," American Control Conference, IEEE, 2014.

${ }^{8}$ Megretski, A., "KYP Lemma for Non-Strict Inequalities and the associated Minimax Theorem," arXic, 2010. 
${ }^{9}$ Pfifer, H. and Seiler, P., "Robustness Analysis of Linear Parameter Varying Systems Using Integral Quadratic Constraints," International Journal of Robust and Nonlinear Control, 2014.

${ }^{10}$ Seiler, P., "Nonlinear Stability Analysis with Dissipation Inequalities and Integral Quadratic Constraints," accepted to the IEEE Transactions on Automatic Control, 2014

${ }^{11}$ Zames, G. and Falb, P., "Stability Conditions for Systems with Monotone and Slope-Restricted Nonlinearities," SIAM Journal on Control, Vol. 6, No. 1, 1968, pp. 89-108.

${ }^{12} \mathrm{Wu}, \mathrm{F}$., Yang, X. H., Packard, A., and Becker, G., "Induced $\mathcal{L}_{2}$-Norm Control for LPV Systems with Bounded Parameter Variation Rates," International Journal of Robust and Nonlinear Control, Vol. 6, 1996, pp. 983 - 998.

${ }^{13}$ Knoblach, A., Assanimoghaddam, M., Pfifer, H., and Saupe, F., "Robust Performance Analysis: a Review of Techniques for Dealing with Infinite Dimensional LMIs," International Conference on Control Applications Part of the Multi-Conference on Systems and Control, IEEE, 2013.

${ }^{14}$ Hindi, H. and Boyd, S., "Analysis of Linear Systems with Saturation using Convex Optimization," Conference on Decision and Control, IEEE, 1998.

${ }^{15} \mathrm{Hu}, \mathrm{T} .$, Lin, Z., and Chen, B. M., "An analysis and design method for linear systems subject to actuator saturation and disturbance," Automatica, Vol. 38, 2002, pp. 351 - 359.

${ }^{16} \mathrm{Hu}, \mathrm{T}$. and Lin, Z., "Exact Characterization of Invariant Ellipsoids for Single Input Linear Systems Subject to Actuator Saturation," IEEE Transactions on Automatic Control, Vol. 47, No. 1, 2002, pp. 164-169.

${ }^{17}$ Summers, E. and Packard, A., " $\mathcal{L}_{2}$ Gain Verification for Interconnections of Locally Stable Systems Using Integral Quadratic Constraints," Conference on Decision and Control, IEEE, 2010.

${ }^{18}$ Bisplinghoff, R., Ashley, H., and Halfman, R., Aeroelasticity, Dover Pubns, 1955.

${ }^{19}$ Apkarian, P. and Noll, D., "Nonsmooth $\mathcal{H}_{\infty}$ Synthesis," IEEE Transactions on Automatic Control, Vol. 51, No. 1, 2006, pp. $71-86$. 